\title{
Relação entre Apego e Obesidade: Revisão Sistemática da Literatura
}

\author{
Suélen Henriques da Cruz \\ Regina Basso Zanon \\ Cleonice Alves Bosa \\ Universidade Federal do Rio Grande do Sul \\ Porto Alegre, RS, Brasil
}

\begin{abstract}
RESUMO
Além de dieta inadequada e sedentarismo, fatores psicológicos podem estar envolvidos na etiologia da obesidade, incluindo incapacidade de controlar emoções que surgem em momentos de estresse. Nesse caso, o ato de comer se torna uma forma de coping contra as emoções negativas. Esse estudo objetivou investigar a associação entre apego e obesidade, por meio de uma revisão sistemática da literatura, realizada em Julho de 2014, sem limitação de tempo, nas bases de dados eletrônicas PubMed, Web of Science, PsycINFO e LILACS. Foram incluídos no estudo 16 artigos, e a relação entre apego inseguro e obesidade foi mencionada por todos eles. A Teoria do Apego pode ser uma abordagem particularmente útil para o entendimento do "comer emocional" e da obesidade.
\end{abstract}

Palavras-chave: Apego; Obesidade; Revisão sistemática.

\begin{abstract}
Relationship between Attachment and Obesity: A Systematic Literature Review

Besides inappropriate diet and lack of physical activity, psychological factors may be involved in the etiology of obesity, including the inability to control emotions that arise at stressful moments. In this case, eating becomes a way to cope with negative feelings. This study aimed to investigate the association between attachment and obesity through a systematic literature review, carried out in July 2014, with no time limitation, in the electronic databases PubMed, Web of Science, PsycINFO and LILACS. Sixteen articles were included in this study and all of them mentioned the relationship between insecure attachment and obesity. Attachment Theory seems to be a particularly useful approach in the understanding of emotional eating and obesity.
\end{abstract}

Keywords: Attachment; Obesity; Systematic review.

\section{RESUMEN}

\section{La Relación entre Apego y Obesidad: Una Revisión Sistemática de la Literatura}

Allá mala alimentación y inactividad física, factores psicológicos pueden estar implicados en la etiología de la obesidad, incluyendo la incapacidad para controlar las emociones que surgen en momentos de estrés. Neste caso el acto de comer se torna una manera de lidiar con las emociones negativas. El objetivo de este estúdio fué investigar la asociación entre apego y obesidad, a través de una revisión sistemática de la literatura que se realizó en julio de 2014 y sin limitación de tiempo, en las bases de datos electrónicas PubMed, Web of Science, PsycINFO y LILACS. Fueron incluidos no estudio 16 artículos y la relación entre apego inseguro y obesidade fué mencionada por todos ellos. La Teoría del Apego es particularmente útil para la comprensión del "comer emocional” y de la obesidad.

Palabras clave: Apego; Obesidad; Revisión sistemática. 


\section{INTRODUÇÃO}

Devido ao aumento alarmante em sua prevalência, a obesidade tem sido reconhecida mundialmente como um importante desafio para a saúde pública, ao afetar cerca de 500 milhões de adultos no mundo (OMS, 2012). A obesidade acarreta diversos prejuízos à saúde, de modo que ter um índice de massa corporal (IMC) acima do recomendado para a faixa etária constitui um dos maiores fatores de risco para diversas doenças, dentre elas as cardiovasculares, o diabetes tipo II e vários tipos de câncer (Prentice \& Viner, 2013; OMS, 2009). Além disso, a obesidade também pode ocasionar problemas emocionais, tais como baixa autoestima, depressão e ansiedade (Kim \& Park, 2009; Vila et al., 2004), além de funcionamento social empobrecido e a dificuldade de relacionamento (Pitrou, Shojaei, Wazana, Gilbert, \& Kovess-Masféty, 2010).

As causas da obesidade tem sido atribuídas, nas sociedades ocidentais, a estilos de vida pouco saudáveis, caracterizados pelo consumo de alimentos altamente calóricos, e pela diminuição na prática de atividade física, tão comuns na atualidade (Pereira, Francischi, \& Lancha Jr, 2003; Ribas \& Silva, 2014). Sendo assim, os programas que buscam prevenir a obesidade e/ou estimular o emagrecimento, em sua maioria, se concentram em promover atividade física e o consumo de alimentos saudáveis (OMS, 2009). Entretanto, nem sempre esses programas obtém sucesso, pois a obesidade é uma condição multicausal, que envolve em sua etiologia não só fatores comportamentais, mas também genéticos, metabólicos, sociais, culturais e psicológicos (Karnik \& Karnekar, 2012).

A respeito dos fatores psicológicos envolvidos na etiologia da obesidade, alguns estudos sugerem que indivíduos podem comer em excesso devido a um descontrole emocional (Nguyen-Rodriguez, Unger, \& Spruijt-Metz, 2009), ou seja, uma incapacidade de regular e controlar as emoções que surgem em momentos de estresse psicológico (Thompson, 1994; Wansink \& Payne, 2007). A regulação emocional é uma habilidade de autorregulação que se desenvolve na infância, e controla a impulsividade e os processos afetivos ligados à alimentação, regulando de forma automática a ingestão de alimentos em resposta a sinais fisiológicos de fome e saciedade (Puder \& Munsch, 2010; Johnson \& Birch, 1994). Entretanto, esses sinais podem ser influenciados por experiências precoces negativas e por fatores emocionais e sociais (Graziano, Calkins, \& Keane, 2010), de tal forma que se pode consumir excessivamente alimentos mesmo na ausência de fome, com o intuito de regular e reduzir emoções negativas como tristeza e ansiedade
(Braet, 2005; Goossens, Braet, Van Vlierberghe, \& Mels, 2008). Esse processo é denominado "comer emocionalmente" [emotional eating] (Graziano et al., 2010) e, de acordo com essa perspectiva, a obesidade pode ter início na dificuldade do indivíduo em regular suas emoções, resultando o ato de comer em excesso numa forma de coping contra afetos negativos (Pervanidou \& Chrousus, 2011).

Dentre as abordagens teóricas utilizadas no entendimento da relação entre emoções e obesidade destaca-se a Teoria do Apego, de Bowlby (1982). De acordo com essa teoria, os seres humanos nascem com um sistema psicobiológico e adaptativo que os motiva a buscar proximidade de pessoas significativas (figuras de apego) nos momentos em que se sentem ameaçados ou precisam de ajuda. Interações com figuras de apego disponíveis, sensíveis e responsivas às necessidades de proximidade e suporte promovem um senso de segurança, gerando representações mentais positivas do Self e dos outros. Ao contrário, quando as figuras de apego são indisponíveis, o desenvolvimento psíquico é prejudicado, havendo redução na resiliência e na capacidade de coping em momentos estressantes, predispondo o indivíduo a dificuldades de lidar com crises (Bowlby, 1988).

Nesse sentido, interações com figuras de apego estáveis e o sentimento de segurança resultante delas promovem suporte real e simbólico para a aprendizagem de estratégias de autorregulação (Mikulincer \& Shaver, 2012). Segundo Cassidy (1994), essas interações propiciam às crianças aprender que é socialmente aceitável expressar suas emoções, bem como tentar entender os sentimentos dos outros. A segurança no apego, portanto, reflete o desenvolvimento da capacidade de autorregulação emocional e o fornecimento de respostas adequadas ao estresse. Em contrapartida, a insegurança no apego pode ser um fator de risco para obesidade (Anderson \& Whitaker, 2011), já que a capacidade de autorregulação é prejudicada e o ato de comer é utilizado como única estratégia viável para aplacar a angústia gerada pelos conflitos emocionais.

Tendo em vista que a obesidade é uma condição multicausal, estudar também os aspectos emocionais associados a essa condição proporciona uma visão mais ampla sobre as causas do excesso de peso, oportunizando, dessa forma, a construção de estratégias de prevenção e tratamento que vão além da dieta e da prática de atividade física. Nesse sentido, a Teoria do Apego, ao destacar a importância das relações iniciais entre a criança e seus principais cuidadores para a regulação das emoções e a autoestima (Bowlby, 1982; Levitan \& Davis, 2010), oferece um aporte teórico consistente para o entendimento da relação entre 
problemas emocionais e obesidade. Dessa forma, o conhecimento acerca da qualidade da relação inicial entre a criança e seu principal cuidador pode não só embasar estratégias para prevenir o excesso de peso, mas também outras formas de transtornos emocionais futuros. Com base no exposto, o objetivo do presente artigo foi investigar a relação entre apego e obesidade. Para tanto, realizou-se um estudo de revisão sistemática da literatura, visando identificar as principais contribuições acerca desta temática, bem como destacar áreas que necessitam de um maior investimento empírico.

\section{MÉTODO}

Procedeu-se, inicialmente, uma busca de artigos indexados nas bases de dados eletrônicas PubMed, Web of Science, PsycINFO e LILACS. Para isso, foram realizadas seis combinações de descritores, dois a dois, em cada uma das bases selecionadas: "attachment" e "obesity"; "attachment" e "overweight"; "attachment" e "body weight"; "attachment" e "overeating"; "attachment" e "disinhibited eating"; e "attachment" e "weight gain". Com base na análise dos resumos, foram selecionados somente estudos que atenderam aos seguintes critérios de inclusão: (1) foram publicados na modalidade de artigo científico; (2) investigaram especificamente a relação entre apego e obesidade e/ou sobrepeso; (3) eram artigos empíricos; (4) estavam escritos em língua portuguesa, inglesa ou espanhola; e (5) os participantes eram seres humanos.

Foram incluídos na análise tanto os estudos que investigaram o estilo de apego em indivíduos obesos (crianças, adolescentes ou adultos) quanto os que avaliaram essa variável em pais de crianças obesas. mais, pois costumam apresentar níveis maiores de psicopatologia (Braet \& Marvielde, 1997). Isso poderia igualmente enviesar o estudo e, por esse motivo, os artigos que possuem tais indivíduos em suas amostras foram excluídos.

As buscas foram realizadas durante o mês de julho de 2014 e não foi estabelecido limite de data para as publicações. Os resumos repetidos nas bases de dados foram excluídos e, por fim, uma análise minuciosa, na íntegra, dos artigos que atenderam aos critérios de inclusão foi realizada a fim de se definir o número final de estudos a serem revisados. A seleção dos artigos e a extração dos dados foram feitas por dois avaliadores de forma independente. Quando os dois juízes não entraram em consenso em relação ao número de artigos selecionados no estudo, um terceiro juiz foi consultado. No caso de o artigo não estar disponível na íntegra nas bases de dados selecionadas, este foi solicitado diretamente aos autores que, em sua maioria, disponibilizaram prontamente suas publicações.

Nas buscas realizadas, foram encontrados 1253 artigos, sendo 628 na base de dados PubMed, 478 na Web of Science, 140 na PsycINFO e sete na LILACS. A partir da leitura do título e do resumo dos mesmos, 40 estudos foram selecionados por atenderem aos critérios de inclusão previamente estabelecidos. Destes, 21 foram excluídos por serem repetidos e, após a leitura na íntegra, mais três estudos foram retirados da amostra por não terem investigado especificamente as variáveis de interesse. Sendo assim, a amostra desse estudo resultou em 16 artigos. O fluxograma do processo de obtenção dos artigos incluídos na presente revisão pode ser visualizado na Figura 1.

Ademais, foram excluídos os resumos de pesquisas envolvendo participantes com transtornos alimentares (e.g., anorexia, bulimia), bem como aqueles que investigaram a influência do estilo de apego na adesão a programas de intervenção para redução de peso. A exclusão dos estudos sobre transtornos alimentares se deu porque essas condições, por si só, já se constituem em psicopatologia, o que possibilitaria a introdução de vieses na relação entre apego e obesidade. Da mesma forma, pessoas obesas que procuram tratamento tendem a ser diferentes das de-

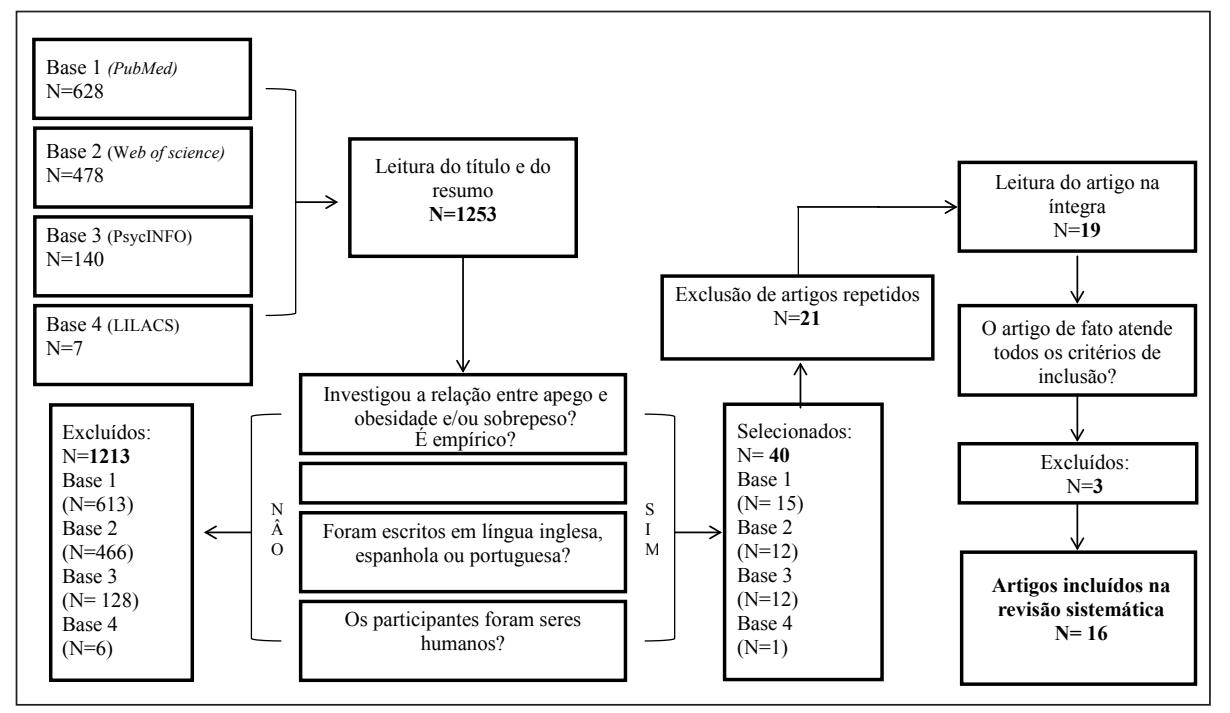

Figura 1. Fluxograma do processo de obtenção dos artigos selecionados para a revisão sistemática 


\section{RESULTADOS}

\section{Caracterização dos estudos}

As principais informações acerca dos estudos incluídos na presente revisão são disponibilizadas na Tabela 1. As referências completas de todos os artigos revisados são apresentadas com asterisco (*) na lista de referências. ${ }^{1}$

O estudo mais antigo sobre a relação entre apego e obesidade foi desenvolvido na Itália e publicado em 2003 (Trombini, Baldkro, Bertaccini, Mattei, Montebarocci, \& Ross, 2003). Foram investigados os estilos de apego e as atitudes emocionais de mães de 30 crianças obesas e 80 com peso dentro da normalidade (eutróficas). Entretanto, foi somente seis anos após sua divulgação que outra pesquisa sobre o assunto foi publicada, desenvolvida igualmente na Itália (D’Argenio, Mazzi, Pecchioli, Di Lorenzo, Siracusano, \& Troisi, 2009). De modo geral, observou-se um aumento da produção científica sobre a relação entre apego e obesidade a partir do ano de 2010, uma vez que somente nos últimos cinco anos foram publicados 14 artigos sobre a temática, representado $87,5 \%$ do total.

TABELA 1

Informações acerca dos estudos incluídos na revisão sistemática, por ordem alfabética

\begin{tabular}{|c|c|c|c|c|c|c|c|}
\hline \multirow{2}{*}{\multicolumn{2}{|c|}{ Estudo }} & \multicolumn{3}{|c|}{ Amostra } & \multirow{2}{*}{$\begin{array}{l}\text { Instrumentos para } \\
\text { avaliação do apego }\end{array}$} & \multirow{2}{*}{$\begin{array}{l}\text { Métodos } \\
\text { de análise }\end{array}$} & \multirow{2}{*}{ Principais achados } \\
\hline & & $N$ & Idade & Sexo & & & \\
\hline 1 & $\begin{array}{l}\text { Alexander, K. E., \& Siegel, H. I. (2013). } \\
\text { Perceived hunger mediates the relationship } \\
\text { between attachment anxiety and emotional } \\
\text { eating. Eating Behaviors, 14, 374-377. }\end{array}$ & 97 & $\begin{array}{l}\text { Média: } \\
20.69 \text { anos }\end{array}$ & $\begin{array}{l}\text { Feminino e } \\
\text { masculino }\end{array}$ & $\begin{array}{l}\text { Experiences in } \\
\text { Close Relationships } \\
\text { Questionnaire- } \\
\text { Revised }\end{array}$ & $\begin{array}{l}\text { Correlação de } \\
\text { Pearson }\end{array}$ & $\begin{array}{l}\text { Correlação significativa } \\
\text { entre apego inseguro e } \\
\text { IMC. }\end{array}$ \\
\hline 2 & $\begin{array}{l}\text { Anderson, S. E., Gooze, R. A., Lemeshow, } \\
\text { S., \& Whitaker, R. C. (2012). Quality of } \\
\text { early maternal-child relationship and risk } \\
\text { of adolescent obesity. Pediatrics, } 129(1) \text {, } \\
\text { 131-141. }\end{array}$ & 977 & $\begin{array}{l}\text { Entre } 15 \mathrm{e} \\
36 \text { meses }\end{array}$ & $\begin{array}{l}\text { Feminino e } \\
\text { masculino }\end{array}$ & $\begin{array}{l}\text { Observações da } \\
\text { interação mãe- } \\
\text { criança } \\
\text { Toddler Attachment } \\
\text { Q-sort }\end{array}$ & $\begin{array}{l}\text { Regressão } \\
\text { logística }\end{array}$ & $\begin{array}{l}\text { Apego inseguro, aos } 24 \\
\text { meses, associou-se com o } \\
\text { aumento da probabilidade } \\
\text { de obesidade na } \\
\text { adolescência. }\end{array}$ \\
\hline 3 & $\begin{array}{l}\text { Anderson, S. E., \& Whitaker, R. C. } \\
\text { (2011). Attachment security and obesity } \\
\text { in us preschool-aged children. Archives of } \\
\text { Pediatrics \& Adolescent Medicine, 165(3), } \\
\text { 235-242. }\end{array}$ & 6,650 & $\begin{array}{l}\text { Entre } 24.3 \text { e } \\
54.3 \text { meses }\end{array}$ & $\begin{array}{l}\text { Feminino e } \\
\text { masculino }\end{array}$ & $\begin{array}{l}\text { Toddler Attachment } \\
\text { Q-sort }\end{array}$ & $\begin{array}{l}\text { Regressão } \\
\text { logística }\end{array}$ & $\begin{array}{l}\text { Obesidade associou-se ao } \\
\text { apego inseguro. }\end{array}$ \\
\hline 4 & $\begin{array}{l}\text { Bahrami, F., Kelishadi, R., Jafari, N., Kaveh, } \\
\text { Z., \& Isanejad, O. (2013). Association } \\
\text { of children's obesity with the quality of } \\
\text { parental-child attachment and psychological } \\
\text { variables. Acta Pcediatrica, 102, 321-324. }\end{array}$ & 202 & $\begin{array}{l}\text { Entre } 9 \text { e } \\
13 \text { anos }\end{array}$ & $\begin{array}{l}\text { Feminino e } \\
\text { masculino }\end{array}$ & $\begin{array}{l}\text { The Inventory of } \\
\text { Parent and Peer } \\
\text { Attachment-Revised } \\
\text { Version for Children }\end{array}$ & $\begin{array}{l}\text { Modelo de } \\
\text { equações } \\
\text { estruturais }\end{array}$ & $\begin{array}{l}\text { Correlação significativa } \\
\text { e negativa entre IMC e } \\
\text { qualidade do apego. }\end{array}$ \\
\hline 5 & $\begin{array}{l}\text { Cooper, M. J., \& Warren, L. (2011). The } \\
\text { relationship between body weight (body } \\
\text { mass index) and attachment history in young } \\
\text { women. Eating Behaviors, } 12(1), 94-96 \text {. }\end{array}$ & 145 & $\begin{array}{l}\text { Média: } \\
24.32 \text { anos }\end{array}$ & Feminino & $\begin{array}{l}\text { Attachment History } \\
\text { Questionnaire }\end{array}$ & $\begin{array}{l}\text { Regressão } \\
\text { múltipla }\end{array}$ & $\begin{array}{l}\text { O estilo parental relacionou- } \\
\text { se com o alto peso (IMC) } \\
\text { da criança. }\end{array}$ \\
\hline 6 & $\begin{array}{l}\text { D'Argenio, A., Mazzi, C., Pecchioli, L., } \\
\text { Di Lorenzo, G., Siracusano A. \& Troisi, A. } \\
\text { (2009). Early trauma and adult obesity: is } \\
\text { psychological dysfunction the mediating } \\
\text { mechanism? Physiology \& Behavior, 98, } \\
\text { 543-546. }\end{array}$ & 200 & $\begin{array}{l}\text { Média: } \\
40.38 \text { anos }\end{array}$ & $\begin{array}{l}\text { Feminino e } \\
\text { masculino }\end{array}$ & $\begin{array}{l}\text { Relationship } \\
\text { Questionnaire }\end{array}$ & $\begin{array}{l}\text { Regressão } \\
\text { logística binária }\end{array}$ & $\begin{array}{l}\text { Alto IMC associou-se ao } \\
\text { apego do tipo inseguro- } \\
\text { ansioso. }\end{array}$ \\
\hline 7 & $\begin{array}{l}\text { Goossens, L., Braet, C., Bosmans, G., \& } \\
\text { Decaluwé, V. (2011). Loss of control over } \\
\text { eating in pre-adolescent youth: the role } \\
\text { of attachment and self-esteem. Eating } \\
\text { Behaviors, 12(4), 289-295. }\end{array}$ & 555 & $\begin{array}{l}\text { Média: } \\
9.02 \text { anos }\end{array}$ & $\begin{array}{l}\text { Feminino e } \\
\text { masculino }\end{array}$ & Security Scale & $\begin{array}{l}\text { Regressão } \\
\text { logística }\end{array}$ & $\begin{array}{l}\text { Grupo de crianças com } \\
\text { comer compulsivo } \\
\text { apresentou menor índice } \\
\text { de apego seguro em } \\
\text { relação a mãe e ao pai, em } \\
\text { comparação com o grupo de } \\
\text { crianças sem esse quadro. }\end{array}$ \\
\hline 8 & $\begin{array}{l}\text { Goossens, L., Braet, C., Van Durmea, K., } \\
\text { Decaluwé, V., \& Bosmans, G. (2012). } \\
\text { The parent-child relationship as predictor } \\
\text { of eating pathology and weight gain in } \\
\text { preadolescents. Journal of Clinical Child \& }\end{array}$ & 601 & $\begin{array}{l}\text { Média: } \\
9.05 \text { anos }\end{array}$ & $\begin{array}{l}\text { Feminino e } \\
\text { masculino }\end{array}$ & Security Scale & $\begin{array}{l}\text { Correlação de } \\
\text { Pearson e de } \\
\text { Spearman }\end{array}$ & $\begin{array}{l}\text { Apego inseguro com a } \\
\text { mãe foi um fator de risco } \\
\text { para ganho de peso um ano } \\
\text { depois. }\end{array}$ \\
\hline
\end{tabular}

Continua

\footnotetext{
${ }^{1}$ As referências dos artigos não selecionados para a presente revisão sistemática, bem como o motivo da exclusão, poderão ser solicitadas às autoras do estudo.
} 
TABELA 1 - conclusão

\begin{tabular}{|c|c|c|c|c|c|c|c|}
\hline \multirow{2}{*}{\multicolumn{2}{|c|}{ Estudo }} & \multicolumn{3}{|c|}{ Amostra } & \multirow{2}{*}{$\begin{array}{l}\text { Instrumentos para } \\
\text { avaliação do apego }\end{array}$} & \multirow{2}{*}{$\begin{array}{l}\text { Métodos } \\
\text { de análise }\end{array}$} & \multirow{2}{*}{ Principais achados } \\
\hline & & $N$ & Idade & Sexo & & & \\
\hline 9 & $\begin{array}{l}\text { Guzmán, A. T. (2012). Attachment } \\
\text { representation of children with obesity and } \\
\text { the sensitive response of their mothers. } \\
\text { Summa psicológica UST, } 9(2), 57-67 \text {. }\end{array}$ & 16 & $\begin{array}{l}\text { Mães: }<32 \text { anos } \\
\text { Filhos entre } \\
7 \text { e } 12 \text { anos }\end{array}$ & $\begin{array}{l}\text { Feminino e } \\
\text { masculino }\end{array}$ & $\begin{array}{l}\text { Child Attachment } \\
\text { Interview }\end{array}$ & $\begin{array}{l}\text { Qualitativa, } \\
\text { Grounded } \\
\text { Theory }\end{array}$ & $\begin{array}{l}\text { Crianças obesas } \\
\text { apresentaram apego } \\
\text { inseguro. }\end{array}$ \\
\hline 10 & $\begin{array}{l}\text { Hernandez-Hons, A. \& Woolley, S. R. (2012). } \\
\text { Women's Experiences with Emotional Eating } \\
\text { and Related Attachment and Sociocultural } \\
\text { Processes. Journal of Marital and Family } \\
\text { Therapy, 38(4), 589-603. }\end{array}$ & 8 & $\begin{array}{l}\text { Entre } 30 \text { e } \\
62 \text { anos }\end{array}$ & Feminino & $\begin{array}{l}\text { Entrevista } \\
\text { semiestruturada }\end{array}$ & $\begin{array}{l}\text { Análise } \\
\text { fenomenológica }\end{array}$ & $\begin{array}{l}\text { Qualidade da relação } \\
\text { de apego com pessoas } \\
\text { significativas foi semelhante } \\
\text { à relação estabelecida com a } \\
\text { comida e o comer. }\end{array}$ \\
\hline 11 & $\begin{array}{l}\text { Hintsanen, M.; Jokela, M., Pulkki-Raback, } \\
\text { L., Viikari, J. S. A., \& Keltikangas-Järvinen, } \\
\text { L. (2010). Associations of youth and } \\
\text { adulthood body-mass index and waist-hip } \\
\text { ratio with attachment styles and dimensions. } \\
\text { Current Psychology, 29, 257-271. }\end{array}$ & 1,570 & $\begin{array}{l}\text { Entre } 24 \text { e } \\
39 \text { anos }\end{array}$ & $\begin{array}{l}\text { Feminino e } \\
\text { masculino }\end{array}$ & $\begin{array}{l}\text { Relationship } \\
\text { Questionnaire }\end{array}$ & $\begin{array}{l}\text { Regressão } \\
\text { linear }\end{array}$ & $\begin{array}{l}\text { Indices maiores de IMC e } \\
\text { de cintura-quadril foram } \\
\text { associados com } \\
\text { maior estilo de apego } \\
\text { inseguro-medroso. }\end{array}$ \\
\hline 12 & $\begin{array}{l}\text { Holland, S., Dallos, R., \& Olver L. } \\
\text { (2011). An exploration of young women's } \\
\text { experiences of living with excess weight. } \\
\text { Clinical Child Psychology and Psychiatry, } \\
\text { 17(4), 538-552. }\end{array}$ & 8 & $\begin{array}{l}\text { Entre } 13 \text { e } \\
16 \text { anos }\end{array}$ & Feminino & $\begin{array}{l}\text { Child Attachment } \\
\text { Interview }\end{array}$ & $\begin{array}{l}\text { Análise } \\
\text { fenomenológica }\end{array}$ & $\begin{array}{l}\text { Participantes com sobrepeso } \\
\text { adotaram o papel de } \\
\text { cuidadoras em sua família, } \\
\text { geralmente caracterizado } \\
\text { por conflito nas relações } \\
\text { familiares. }\end{array}$ \\
\hline 13 & $\begin{array}{l}\text { Sockalingam, S., Wnuka, S., Strimasa, R., } \\
\text { Hawaa, R., \& Okrainecb, A. (2011). The } \\
\text { association between attachment avoidance } \\
\text { and quality of life in bariatric surgery } \\
\text { candidates. Obesity Facts, 4(6), 456-60. }\end{array}$ & 70 & $\begin{array}{l}\text { Média: } \\
44.26 \text { anos }\end{array}$ & $\begin{array}{l}\text { Feminino e } \\
\text { masculino }\end{array}$ & $\begin{array}{l}\text { Experiences in Close } \\
\text { Relationships Scale }\end{array}$ & $\begin{array}{l}\text { Correlações de } \\
\text { Pearson }\end{array}$ & $\begin{array}{l}\text { Indices maiores de IMC } \\
\text { estiveram associados com } \\
\text { maior estilo de apego } \\
\text { iinseguro-evitativo. }\end{array}$ \\
\hline 14 & $\begin{array}{l}\text { Stenhammar, C., Olsson, G.M., Bahmanyar, } \\
\text { S., Hulting, A-L, Wettergren, B., Edlund, B., } \\
\& \text { Montgomery, S. M. (2010). Family stress } \\
\text { and BMI in young children. Acta Pcediatrica, } \\
\text { 99, 1205-1212. }\end{array}$ & 873 & $\begin{array}{l}0 \text { a } 5 \text { anos do } \\
\text { filho }\end{array}$ & $\begin{array}{l}\text { Feminino e } \\
\text { masculino }\end{array}$ & $\begin{array}{l}\text { Relationship } \\
\text { Questionnaire }\end{array}$ & $\begin{array}{l}\text { Regressão } \\
\text { logística }\end{array}$ & $\begin{array}{l}\text { Associação positiva entre } \\
\text { apego inseguro dos pais e } \\
\text { sobrepeso dos filhos. }\end{array}$ \\
\hline 15 & $\begin{array}{l}\text { Trombini, E., Baldkro, B., Bertaccini, R., } \\
\text { Mattei, C., Montebarocci, O., \& Ross, N. } \\
\text { (2003). Maternal attitudes and attachment } \\
\text { styles in mothers of obese children. } \\
\text { Perceptual and Motor Skills, 97, 613-620. }\end{array}$ & 30 & $\begin{array}{l}\text { Média: } \\
37.4 \text { anos }\end{array}$ & Feminino & $\begin{array}{l}\text { Attachment Style } \\
\text { Questionnaire }\end{array}$ & ANOVA & $\begin{array}{l}\text { Maior prevalência do estilo } \\
\text { de apego inseguro no grupo } \\
\text { de mães de crianças obesas } \\
(66.6 \%)\end{array}$ \\
\hline 16 & $\begin{array}{l}\text { Wilkinson, L. L., Rowe, A. C., Bishop, R. } \\
\text { J., \& Brunstrom, J. M. (2010). Attachment } \\
\text { anxiety, disinhibited eating, and body mass } \\
\text { index in adulthood. International Journal of } \\
\text { Obesity, 34, 1442-1445. }\end{array}$ & 200 & $\begin{array}{l}\text { Média: } 22.4 \\
\text { anos }\end{array}$ & $\begin{array}{l}\text { Feminino e } \\
\text { masculino }\end{array}$ & $\begin{array}{l}\text { Experiences in Close } \\
\text { Relationships }\end{array}$ & $\begin{array}{l}\text { Regressão } \\
\text { múltipla }\end{array}$ & $\begin{array}{l}\text { Correlação significativa } \\
\text { entre IMC e apego do tipo } \\
\text { inseguro-ansioso. }\end{array}$ \\
\hline
\end{tabular}

No que tange às características dos participantes, verificou-se que metade dos estudos $(n=8)$ investigou a relação entre apego e obesidade em adolescentes ou adultos (Alexander \& Siegel, 2013; Cooper \& Warren, 2011; D'Argenio et al., 2009; Holland, Dallos, \& Olver, 2011; Hernandez-Hons \& Woolley, 2012; Hintsanen, Jokela, Pulkki-Raback,Viikari, \& KeltikangasJärvinen, 2010; Sockalingam et. al., 2011; Wilkinson, Rowe, Bishop, \& Brunstrom, 2010), sendo seis pesquisas $(37,5 \%)$ realizadas com crianças (Anderson \& Whitaker, 2011; Anderson, Gooze, Lemeshow, \& Whitaker, 2012; Bahrami, Kelishadi, Jafari, Kaveh, \& Isanejad, 2013; Goossens, Braet, Bosmans, \& Decaluwé, 2011; Goossens, Braet, Durme, Decaluwé \& Bosmans, 2012; Guzmán, 2012). Além disso, foram encontrados dois estudos $(12,5 \%)$ que avaliaram a relação entre o apego dos pais e a obesidade dos filhos (Stenhammar et al., 2010; Trombini et al., 2003).

Quanto ao número de participantes das pesquisas, observou-se uma grande variabilidade, sendo o mínimo de oito (Hernandez-Hons \& Woolley, 2012; Holland, Dallos, \& Olver, 2011) e o máximo de 6650 (Anderson \& Whitaker, 2011), de modo que a maioria das investigações $(56,2 \%, n=9)$ envolveu 200 ou mais participantes. No que se refere ao sexo dos participantes, todos incluíram o sexo feminino, sendo que $75 \%$ dos estudos $(n=12)$ também incluíram sujeitos de sexo masculino. Os instrumentos utilizados para mensurar apego foram diversos e variaram também de acordo com a faixa etária dos participantes. Os dois estudos que investigaram essa variável em bebês (Anderson \& Whitaker, 2011; Anderson et al., 2012), utilizaram a Toddler Attachment Q-sort (AQS), uma escala 
preenchida por um observador treinado, após observar atentamente a relação entre a mãe e a criança. Cabe ressaltar que na pesquisa de Anderson et al. (2012), além da AQS, utilizada quando os bebês tinham 24 meses, foram realizadas também observações da interação mãe-criança, aos 15, 24 e 36 meses de idade, sendo que aos 15 e 36 meses a seguridade do apego foi avaliada em laboratório através do Procedimento da Situação Estranha (Ainsworth, 1979).

Por outro lado, estudos realizados com crianças maiores, com idades variando entre oito e 12 anos (Goossens et al., 2011; Goossens et al., 2012), utilizaram a Security Scale (SS) (Kerns, Klepac, \& Cole, 1996), um questionário de autorrelato que investiga a seguridade das relações entre mãe-criança e pai-criança. Já Holland et al. (2011) e Guzmán (2012) utilizaram a Child Attachment Interview (CAI), uma entrevista constituída por 19 questões, desenvolvidas no Anna Freud Centre (UK; Target, Fonagy, \& Shmueli-Goetz, 2003), que investiga a percepção sobre as figuras de apego disponíveis no momento da entrevista, com base na avaliação da comunicação verbal e não verbal dos participantes.

No que se refere às investigações realizadas com adultos, o Relationship Questionnaire (Bartholomew \& Horowitz, 1991) foi o instrumento mais recorrente (D'Argenio et al., 2009; Hintsanen et al., 2010; Stenhammar et al., 2010). Trata-se de uma medida dos estilos de apego adulto, definidos como inseguro, seguro, preocupado ou evitativo, que leva em consideração os relacionamentos do indivíduo com pessoas em geral, não investigando especificamente as relações amorosas. Destaca-se ainda que HernandezHons e Woolley (2012) investigaram o apego com base em uma entrevista semiestruturada, que está disponível na íntegra no artigo, como Anexo.

A respeito do delineamento dos estudos, a maioria é transversal $(75 \% ; n=12)$, sendo apenas quatro pesquisas de tipo longitudinal (Anderson \& Whitaker, 2011; Anderson et al., 2012; Goossens et al., 2012; Stenhammar et al., 2010). Observou-se um predomínio de estudos quantitativos $(n=12)$, sendo as análises de regressão múltipla ou de regressão logística as mais frequentemente empregadas. Apenas três estudos qualitativos foram realizados e estes empregaram análise interpretativa fenomenológica (Hernandez-Hons \& Woolley, 2012; Holland et al., 2011) e análise guiada pela escola metodológica da Grounded Theory (Guzmán, 2012).

\section{Contribuições empíricas sobre a relação entre apego e obesidade}

Pesquisas com bebês e crianças pequenas demonstram que o apego inseguro, desenvolvido na primeira infância, configura-se como um fator de risco para a obesidade, tanto na infância quanto na adolescência (Anderson \& Whitaker; 2011; Anderson et al., 2012). Em um estudo longitudinal, Anderson e Whitaker (2011) investigaram em 6650 crianças a relação entre o apego seguro aos 24 meses de idade e o risco de desenvolver obesidade aos 4,5 anos. Em crianças com apego inseguro, a prevalência de obesidade aos 4,5 anos de idade foi de $23,1 \%$, comparado com $16.6 \%$ nas crianças com apego seguro. A probabilidade de obesidade aos 4,5 anos entre as crianças com apego inseguro foi de 1.48 (95\% IC: 1.23 , 1.79), ou seja, quase $50 \%$ maior do que a das crianças com apego seguro. Em estudo posterior, Anderson et al. (2012), ao verificarem a correlação entre obesidade e qualidade do relacionamento mãe-criança em 977 díades, encontraram que o apego inseguro, aos 24 meses, esteve associado com o risco de obesidade na adolescência.

Associações entre apego inseguro e ganho de peso também foram relatadas em pesquisas com escolares ou pré-adolescentes (Goossens et al., 2011; Goossens et al., 2012; Guzmán, 2012). Por exemplo, Goossens et al. (2011) analisaram em 555 crianças o papel da autoestima e do tipo apego na explicação da compulsão alimentar, desencadeadora do sobrepeso. Crianças compulsivas relataram significativamente menos apego seguro em relação à mãe e ao pai, em comparação a crianças do grupo controle. De forma semelhante, Goossens et al. (2012) examinaram o papel do apego como preditor do ganho de peso em 601 pré-adolescentes, demostrando que relações inseguras com figura materna estiveram associadas ao ganho de peso um ano depois.

Os resultados das pesquisas realizadas com adolescentes ou adultos também demonstraram relações significativas entre obesidade e a insegurança do apego desenvolvida com figuras de referência (Alexander \& Siegel, 2013; Cooper \& Warren, 2011; D’Argenio et al., 2009). Mais especificamente, foram encontradas correlações significativas entre aumento de IMC e apego do tipo inseguro-ansioso (D'Argenio et al., 2009; Sockalingam et al., 2011; Wilkinson et al., 2010), inseguro-evitativo (Sockalingam et al., 2011) e inseguro-medroso [fearful] (Hintsanen et al., 2010).

Quanto aos estudos que avaliaram o tipo de apego em pais de crianças obesas, destacam-se associações significativas entre apego inseguro dos pais e IMC aumentado nos filhos. Por exemplo, Trombini et al. (2003) buscaram diferenciar mães de crianças obesas de mães de crianças eutróficas através do estilo de apego e das atitudes emocionais. Nesse estudo, houve uma prevalência significativa do estilo de apego inseguro 
no grupo de mães de crianças obesas (66.6\%), ao passo que no grupo de mães de crianças eutróficas, houve maior prevalência do apego de tipo seguro (38.5\%). Stenhammar et al. (2010) confirmaram esses achados em uma pesquisa com 873 crianças, tendo encontrado, entre outros aspectos, associação positiva entre apego inseguro dos pais e sobrepeso dos filhos.

Por outro lado, os estudos que buscaram explorar por meio de análise fenomenológica as experiências de mulheres com excesso de peso mostraram que a obesidade é uma condição complexa e, por isso, requer uma compreensão profunda das relações estabelecidas com outras pessoas e com os alimentos. Holland et al. (2011), por exemplo, encontraram que muitas das participantes com sobrepeso haviam adotado o papel de cuidadoras em sua família, geralmente caracterizado por conflito nas relações familiares. Já HernandezHons e Woolley (2012) concluíram que a qualidade da relação de apego das participantes com pessoas significativas foi semelhante à relação estabelecida com a comida e o comer, uma vez que foi observado, em seus relatos, ambivalência de sentimentos. Para os autores do estudo, o apego inseguro pode estar na base desses sentimentos contraditórios, fazendo com que a comida possa representar, ao mesmo tempo, uma recompensa e uma punição; uma forma de conforto e uma fonte de culpa.

Dois estudos investigaram a diferença entre o tipo de apego desenvolvido com a figura materna e a paterna, separadamente, sugerindo que a relação de apego da criança com a mãe está significativamente relacionada a variáveis envolvidas com a obesidade. Goossens et al. (2011) encontraram que a qualidade da relação entre mãe-criança é mais importante para a associação entre autoestima e comer compulsivo do que a relação pai-criança. Em outro estudo, realizado por Goossens et al. (2012), o apego inseguro desenvolvido com a mãe foi um preditor do aumento das dietas restritivas, das concepções acerca do peso e forma e do IMC dos filhos. Crianças com vínculo inseguro com a mãe apresentaram risco elevado para o ganho de peso, avaliado um ano mais tarde. Por outro lado, não foram encontradas correlações significativas entre o apego com a figura paterna e o IMC das crianças.

Cabe ressaltar que, juntamente à relação entre estilo de apego e obesidade, grande parte das pesquisas avaliaram outras variáveis que influenciam de alguma forma essa relação. De modo geral, os estudos revisados investigaram a influência de características de personalidade, do quadro psicopatológico e dos hábitos de vida na relação entre apego e obesidade. Dentre as variáveis analisadas, podem-se citar: comer compulsivo (Goossens et al., 2011; Wilkinson et al.,
2010); autoestima (Goossens et al., 2011); ansiedade e depressão (Cooper \& Warren, 2012; Sockalingam et al., 2011); estresse parental (Stenhammar et al., 2010) eventos de vida traumáticos (D'Argenio et al., 2009); qualidade de vida física e mental (Sockalingam et al., 2011); tempo médio semanal que a criança assiste TV (Stenhammar et al., 2010); e sensibilidade materna (Anderson et al., 2012).

Em um estudo com 145 mulheres, Cooper e Warren (2012) encontraram que as variáveis ansiedade e depressão mediaram parcialmente a relação entre apego e obesidade. De modo semelhante, Sockalingam et al. (2011) evidenciaram associações significativas entre os sintomas depressivos de 70 pacientes obesos e o tipo de apego ansioso e evitativo, que, por sua vez, foram preditores do IMC. Por outro lado, Anderson et al. (2012) investigaram longitudinalmente a relação entre a qualidade da relação mãe-criança, na infância, e o risco para obesidade na adolescência. Além do tipo de apego, os autores avaliaram também o papel preditivo da sensibilidade materna no IMC. Nesse estudo, a baixa sensibilidade materna esteve mais fortemente relacionada ao risco de obesidade do que o apego inseguro, configurando-se como preditor do IMC em todos os períodos analisados - 15, 24 e 36 meses. Outra característica parental avaliada, também associada com o IMC de crianças, foi o nível de estresse, especialmente o materno (Stenhammar et al., 2010).

A análise da influência da qualidade de vida física e mental no tipo de apego e no IMC foi realizada por Sockalingam et al. (2011), que encontraram correlações negativas entre a qualidade de vida mental e o apego do tipo ansioso e evitativo, igualmente associados com o IMC. A qualidade de vida física também esteve significativa e negativamente correlacionada com o IMC. A relação entre sobrepeso e assistência à televisão foi encontrada por Stenhammar et al. (2010), de forma que quanto maior é o tempo médio semanal em que as crianças passam assistindo à $\mathrm{TV}$, maior é o sobrepeso das mesmas.

\section{DISCUSSÃO}

A relação entre estilo de apego inseguro e obesidade foi demonstrada por todos os estudos incluídos nessa revisão, indicando que as relações estabelecidas entre o indivíduo e sua figura principal de apego são de fundamental importância para seu desenvolvimento, incluindo questões relacionadas ao peso. $\mathrm{O}$ apego inseguro foi não só mais prevalente em indivíduos obesos, corroborando a relação entre apego e obesidade, mas constituiu preditor significativo de ganho de peso 
em crianças e adolescentes. A Teoria do Apego sugere que, quando os pais não conseguem transmitir aos filhos a segurança e a confiança de que podem contar com seu apoio em momentos de necessidade, as crianças podem se tornar menos capazes de regular as emoções e a impulsividade o que, em alguns casos, pode acabar resultando em obesidade. Nesses casos, o mecanismos de autorregulação emocional se mostram falhos, e os indivíduos passam a comer como forma de lidar com sentimentos negativos, que não conseguem controlar. O alimento, assim, passa a servir como alívio para os momentos estressantes e provocadores de ansiedade, se tornando uma espécie de conforto diante das situações difíceis de serem manejada (Pervanidou \& Chrousus, 2011).

Entre os estudos que investigaram se há diferença entre o tipo de apego desenvolvido com a figura materna e a paterna no que se refere à obesidade dos filhos, foi constatado que somente a qualidade da relação de apego estabelecida com a mãe está associada ao comer compulsivo da criança (Goossens et al., 2011). A esse respeito, pode-se pensar que, na atualidade, embora os homens estejam exercendo papéis desempenhados mais comumente pelas mulheres, como o cuidado dos filhos, por exemplo, as mães ainda são as maiores responsáveis pelas crianças, ao menos enquanto são bebês e dependem mais delas. Assim sendo, mães com problemas psicológicos graves como depressão (Favaro \& Santonastaso, 1995) e estresse (Stenhammar et al., 2010) podem não desempenhar adequadamente a maternagem, tornando-se insensíveis às angústias e necessidades de seus bebês, decorrendo disso o desenvolvimento de um estilo de apego inseguro por parte das crianças que, futuramente, podem buscar preencher o sentimento de vazio proveniente dessa falha na relação com a figura materna com comida (Campo, 2004). Desse modo, ocupar-se da saúde mental das mães, prevenindo a ocorrência de quadros psicopatológicos especialmente nos períodos gestacional e puerperal, quando se estabelece a relação de apego com o bebê, pode impedir a ocorrência de problemas futuros na criança, dentre eles, a impulsividade e o sobrepeso.

Outras variáveis pesquisadas enquanto mediadores da relação entre estilo de apego e ganho de peso corroboram a complexidade da etiologia da obesidade e a importância de se abordar os aspectos emocionais. $\mathrm{O}$ estilo de apego desenvolvido serve como fator estruturante da personalidade do indivíduo (Bowlby,
1982), exercendo papel fundamental no modo como este se comporta diante das mais diversas situações de vida. Sendo assim, a ocorrência de eventos estressantes, como perdas, por exemplo, pode mediar a relação entre o estilo de apego e obesidade, de modo que uma pessoa com apego inseguro, diante de uma perda de um ente querido, pode conseguir aplacar sua dor apenas através do consumo alimentar exagerado. Por outro lado, o apego ansioso e o consequente "comer emocional" também pode ser mediado pela incapacidade de o indivíduo distinguir entre fome e estresse (Alexander \& Siegel, 2013). Segundo essa perspectiva, o indivíduo reage a situações estressantes comendo, pois fisiologicamente, confunde essas duas sensações.

Na realização do presente trabalho, além de terem sido encontrados poucos estudos que abordam a relação entre apego e obesidade, observou-se grande diversidade de algumas características metodológicas, especialmente em relação à amostra e aos instrumentos utilizados, o que dificulta a comparação dos resultados. Somado a isso, ressalta-se a carência de estudos longitudinais e com análises qualitativas. No caso da relação entre apego e obesidade, estudos longitudinais podem apontar se o tipo de apego estabelecido contribui para o desenvolvimento de obesidade ao longo do tempo, por exemplo. Da mesma forma, estudos qualitativos permitem estudar a temática em maior profundidade e, nesse sentido, poderiam explicar melhor a relação entre estilos de apego e obesidade. Cumpriu-se o objetivo de investigar a associação entre apego e obesidade por meio de uma revisão sistemática da literatura existente, contudo, este trabalho teve a limitação de não avaliar o rigor metodológico das pesquisas revisadas, o que permitiria uma maior confiabilidade na interpretação dos resultados aqui apresentados.

\section{CONCLUSÃO}

A partir desse estudo, verificou-se que as pesquisas revisadas encontraram associações importantes entre apego inseguro e obesidade, ressaltando que fatores emocionais também estão implicados na etiologia e manutenção do excesso de peso. $\mathrm{O}$ conhecimento acerca destes aspectos pode embasar estratégias mais efetivas de prevenção e tratamento da obesidade, que considerem, além das características biológicas e dos comportamentos, fatores psicológicos, como a habilidade de autorregulação emocional. 


\section{REFERÊNCIAS}

Ainsworth, M. S. (1979). Infant-mother attachment. American Psychologist, 34(10), 932-937. http://dx.doi. org/10.1037/0003-066X.34.10.932

* Alexander, K. E. \& Siegel, H. I. (2013). Perceived hunger mediates the relationship between attachment anxiety and emotional eating. Eating Behaviors, 14, 374-377. http://dx.doi.org/10.1016/j.eatbeh.2013.02.005

* Anderson, S. E. \& Whitaker, R. C. (2011). Attachment security and obesity in us preschool-aged children. Archives of Pediatrics \& Adolescent Medicine, 165(3), 235-242. http://dx.doi.org/10.1001/archpediatrics.2010.292

* Anderson, S. E., Gooze, R. A., Lemeshow, S., \& Whitaker, R. C. (2012). Quality of early maternal-child relationship and risk of adolescent obesity. Pediatrics, 129(1), 131-141. http://dx.doi.org/10.1542/peds.2011-0972

* Bahrami, F., Kelishadi, R., Jafari, N., Kaveh, Z., \& Isanejad, O. (2013). Association of children's obesity with the quality of parental-child attachment and psychological variables. Acta Pcediatrica, 102, 321-324. http://dx.doi.org/10.1111/ apa. 12253

Bartholomew, K. \& Horowitz, L. M. (1991). Attachment styles among young adults: a test of a four-category model. Journal of Personality and Social Psychology, 61(2), 226-244. http://dx.doi.org/10.1037/0022-3514.61.2.226

Bowlby, J. (1982). Attachment Volume 1: Attachment and Loss (2nd ed.). London: Hogarth Press.

Bowlby, J. (1988). A secure base: clinical applications of attachment theory. London: Routledge.

Braet, C. (2005). Psychological profile to become and to stay obese. International Journal of Obesity, 29, 19-23. http:// dx.doi.org/10.1038/sj.ijo.0803100

Braet, C. \& Marvielde, I. (1997). Psychological aspects of childhood obesity: a controlled study in a clinical and nonclinical sample. Journal of Pediatric Psychology, 22(1), 59-71. http://dx.doi.org/10.1093/jpepsy/22.1.59

Campos, A. L. R. (2004). Aspectos psicológicos da obesidade. In M. Fisberg (Ed.). Atualização em obesidade na infância (pp. 97-102). São Paulo: Atheneu.

Cassidy, J. (1994). Emotion regulation: Influences of attachment relationships. Monographs of the Society for Research in Child Development, 59, 228-83. http://dx.doi.org/10.2307/1166148

* Cooper, M. J. \& Warren, L. (2011). The relationship between body weight (body mass index) and attachment history in young women. Eating Behaviors, 12(1), 94-96. http://dx.doi.org/10.1016/j.eatbeh.2010.11.006

* D’Argenio, A., Mazzi, C., Pecchioli, L., Di Lorenzo, G., Siracusano, A. \& Troisi, A. (2009). Early trauma and adult obesity: is psychological dysfunction the mediating mechanism? Physiology \& Behavior, 98, 543-546. http://dx.doi. org/10.1016/j.physbeh.2009.08.010

El-Sayed, A. M., Scarborough, P., \& Galea, S. (2012). Socioeconomic inequalities in childhood obesity in the United Kingdom: A systematic review of the literature. Obesity Facts, 65(5), 671-692. http://dx.doi.org/10.1159/000343611

Ercan, S., Dallar, Y. B., Onen, S., \& Engiz, O. (2012). Prevalence of obesity and associated risk factors among adolescents in Ankara, Turkey. Journal of Clinic Research on Pediatric Endocrinology, 4(4), 204-207.

Favaro, A. \& Santonastaso, P. (1995). Effects of parents' psychological characteristics and eating behavior on childhood obesity and dietary compliance. Journal of Psychosomatic Research, 39, 145-151. http://dx.doi.org/10.1016/00223999(94)00097-O

Goossens, L., Braet, C., Van Vlierberghe, L., \& Mels, S. (2008). Loss of control over eating in overweight youngsters: The role of anxiety, depression and emotional eating. European Eating Disorders Review, 17(1), 68-78. http://dx.doi. org/10.1002/erv.892

* Goossens, L., Braet, C., Bosmans, G., \& Decaluwé, V. (2011). Loss of control over eating in pre-adolescent youth: the role of attachment and self-esteem. Eating Behaviors, 12(4), 289-295. http://dx.doi.org/10.1016/j.eatbeh.2011.07.005

* Goossens, L., Braet, C., Van Durmea, K., Decaluwé, V., \& Bosmans, G. (2012). The parent-child relationship as predictor of eating pathology and weight gain in preadolescents. Journal of Clinical Child \& Adolescent Psychology, 41(4), 1-13. http://dx.doi.org/10.1080/15374416.2012.660690

Graziano, P. A. Calkins, S. D., \& Keane, S. P. (2010). Toddler self-regulation skills predict risk for pediatric obesity. International Journal of obesity, 34, 633-641. http://dx.doi.org/10.1038/ijo.2009.288

Guzmán, A. T. (2012). Attachment representation of children with obesity and the sensitive response of their mothers. Summa psicológica UST, 9(2), 57-67.

*Hernandez-Hons, A. \& Woolley, S. R. (2012). Women's Experiences with Emotional Eating and Related Attachment and Sociocultural Processes. Journal of Marital and Family Therapy, 38(4), 589-603. http://dx.doi.org/10.1111/j.17520606.2011.00239.x

*Hintsanen, M., Jokela, M., Pulkki-Raback, L., Viikari, J. S. A., \& Keltikangas-Järvinen, L. (2010). Associations of youth and adulthood body-mass index and waist-hip ratio with attachment styles and dimensions. Current Psychology, 29, 257-271. http://dx.doi.org/10.1007/s12144-010-9084-8

* Holland, S., Dallos, R., \& Olver L. (2011). An exploration of young women's experiences of living with excess weight. Clinical Child Psychology and Psychiatry, 17(4), 538-552. http://dx.doi.org/10.1177/1359104511426411

Johnson, S. L. \& Birch, L. L. (1994). Parent's and children's adiposity and eating style. Pediatrics, 94(5), 653-661.

Karnik, S., \& Karnekar, A. (2012). Childhood obesity: a global public health crisis. International Journal of Preventive Medicine, 3, 1-7.

Kerns, K. A., Klepac, L., \& Cole, A. (1996). Peer relationships and preadolescents' perceptions of security in the childmother relationship. Developmental psychology, 32(3), 457-466. http://dx.doi.org/10.1037/0012-1649.32.3.457 
Kim, B. \& Park, M. J. (2009). The influence of weight and height status on psychological problems of elementary schoolchildren through child behavior checklist analysis. Yonsei Medical Journal, 50(3), 340-344. http://dx.doi. org/10.3349/ymj.2009.50.3.340

Levitan, R. D. \& Davis, C. (2010). Emotions and eating behavior: Implications for the current obesity epidemic. University of Toronto Quarterly, 79(2), 783-799. http://dx.doi.org/10.3138/utq.79.2.783

Mikulincer, M. \& Shaver, P. R. (2012). An attachment perspective on psychopathology. World Psychiatry, 11, 11-15. http://dx.doi.org/10.1016/j.wpsyc.2012.01.003

Nguyen-Rodriguez, S. T., Unger, J. B., \& Spruijt-Metz, D. (2009). Psychological determinants of emotional eating in adolescence. Eating Disorders, 17(3), 211-224. http://dx.doi.org/10.1080/10640260902848543

Organização Mundial de Saúde. (2012). Priorizando áreas para ação no campo da prevenção populacional à obesidade infantil. Acessado em 09/10/2012, em http://www.who.int/dietphysicalactivity/childhood/tools/en/index.html

Organização Mundial de Saúde. (2009). Estratégias de prevenção populacional para obesidade infantil. Acessado em 10/10/2012, em http://www.who.int/dietphysicalactivity/childhood/report/en/index.html.

Pereira, L. O., Francischi, R. P. D., \& Lancha Jr, A. H. (2003). Obesidade: hábitos nutricionais, sedentarismo e resistência à insulina. Arquivos Brasileiros de Endocrinologia e Metabolismo, 47(2), 111-27. http://dx.doi.org/10.1590/S000427302003000200003

Pervanidou, P. \& Chrousus, G. P. (2011). Emotional/Behavioral disorders and obesity in childhood: A clinician's perspective. The European Health Psychologist, 13(3), 48-52.

Pitrou, I., Shojaei, T., Wazana, A., Gilbert, F., \& Kovess-Masféty, V. (2010). Child overweight, associated psychopatology, and social functioning: a french school-based survey in 6- to 11-year-old children. Obesity, 18(4), 809-817. http://dx.doi.org/10.1038/oby.2009.278

Prentice P. \& Viner, R. M. (2013). Pubertal timing and adult obesity and cardio metabolic risk in women and men: a systematic review and meta-analysis. International Journal of Obesity, 37(8), 1036-43. http://dx.doi.org/10.1038/ijo.2012.177

Puder, J. J. \& Munsch, S. (2010). Psychological correlates of childhood obesity. International Journal of Obesity, 34, 37-43. http://dx.doi.org/10.1038/ijo.2010.238

Ribas, S. A. \& da Silva, L. C. S. (2014). Fatores de risco cardiovascular e fatores associados em escolares do Município de Belém, Pará, Brasil. Cadernos de Saúde Pública, 30(3), 577-586. http://dx.doi.org/10.1590/0102-311X00129812

* Sockalingam, S., Wnuka, S., Strimasa, R., Hawaa, R., \& Okrainecb, A. (2011). The association between attachment avoidance and quality of life in bariatric surgery candidates. Obesity Facts, 4(6), 456-60 http://dx.doi.org/ $10.1159 / 000335345$

* Stenhammar, C., Olsson, G.M., Bahmanyar, S., Hulting, A-L, Wettergren, B., Edlund, B., \& Montgomery, S. M. (2010). Family stress and BMI in young children. Acta Pcediatrica, 99, 1205-1212. http://dx.doi.org/10.1111/j.16512227.2010.01776.x

Target, M., Fonagy, P., \& Shmueli-Goetz, Y. (2003). Attachment representations in school-age children: the development of the child attachment interview (CAI). Journal of Child Psychotherapy, 29(2), 171-186. http://dx.doi.org/10.108 0/0075417031000138433

Thompson, R. A. (1994). Emotion regulation: a theme in search of definition. Monographs of the Society for Research in Child Development, 59(2-3), 25-52. http://dx.doi.org/10.2307/1166137

* Trombini, E., Baldkro, B., Bertaccini, R., Mattei, C., Montebarocci, O., \& Ross, N. (2003). Maternal attitudes and attachment styles in mothers of obese children. Perceptual and Motor Skills, 97, 613-620. http://dx.doi.org/10.2466/ pms.2003.97.2.613

Vila, G., Zipper, E., Dabbas, M., Bertrand, C., Robert, J. J., Ricour, C., \& Mouren-Siméoni, M. C. (2004). Mental disorders in obese children and adolescents. Psychosomatic Medicine, 66, 387-394. http://dx.doi.org/10.1097/01. psy.0000126201.12813.eb

*Wilkinson, L. L., Rowe, A. C., Bishop,R. J., \& Brunstrom, J. M. (2010). Attachment anxiety, disinhibited eating, and body mass index in adulthood. International Journal of Obesity, 34, 1442-1445. http://dx.doi.org/10.1038/ijo.2010.72

Zeller, M. H., Reiter-Purtill, J., Modi, A. C., Gutzwiller, J., Vannatta, K., \& Davies, W.H. (2007). Controlled study of critical parent and family factors in the obesogenic environment. Obesity, 15, 126-136. http://dx.doi.org/10.1038/oby.2007.517

\section{Autores:}

SUÉlen HENRIQUES DA CRUZ - Psicóloga pela Universidade Católica de Pelotas, mestre em Epidemiologia pela Universidade Federal de Pelotas e doutoranda em Psicologia pela Universidade Federal do Rio Grande do Sul.

REGINA BASSO ZANON - Psicóloga pela Universidade Regional do Noroeste do Estado do Rio Grande do Sul, mestre e doutoranda em Psicologia pela Universidade Federal do Rio Grande do Sul.

Cleonice Alves Bosa - Psicóloga pela Universidade de Passo Fundo, meste em Psicologia do Desenvolvimento pela Universidade Federal do Rio Grande do Sul e doutora em Psicologia pelo Institute Of Psychiatry. Atualmente é professora adjunta da Universidade Federal do Rio Grande do Sul e coordenadora do Núcleo Integrado de Estudos e Pesquisa em Transtornos do Desenvolvimento (NIEPED).

\section{Endereço para correspondência:}

Suélen Henriques da Cruz

Rua Ramiro Barcelos, 2600 - Térreo

CEP 90035-003 Porto Alegre, RS, Brasil

E-mail: suhcruz@hotmail.com

Recebido em: 28.02 .13

Aceito em: 08.10.14 